\title{
Narrativas de las cuevas de Kumbe Mayo-Perú
}

Artículos originales: ANTROPOLOGÍA

Recibido: 21/04/2021

Aprobado: 12/06/2021

Natali López Aldave

Publicado: 14/10/2021

Universidad Nacional Mayor de San Marcos natialdave@gmail.com

\begin{abstract}
RESUMEN
En el presente artículo, se analizaron cinco cuevas halladas en el centro ceremonial de Kumbe Mayo, ubicado en el departamento de Cajamarca, Perú. El estudio se basó en un análisis espacial e iconográfico y en la revisión de fuentes históricas y etnográficas. La composición de los símbolos hallados en Kumbe Mayo giró en torno a narrativas vinculadas con la sacralidad del agua, el espacio-tiempo, los ancestros y la fertilidad, materializadas en la construcción del canal; así como en las figuras plasmadas en las cuevas, donde destacan los símbolos zoomorfos, geométricos y antropomorfos. Estas narrativas se extendieron a otros sitios de los Andes centrales y de México.
\end{abstract}

Palabras Clave: cuevas, petroglifo, cosmovisión y narrativas.

\section{Narratives of the caves of Kumbe Mayo-Peru}

\begin{abstract}
In this article, five caves found at ceremonial center of Kumbe Mayo, located in the department of Cajamarca, Perú, are analyzed. This study is based on spatial and iconographic analyses, as well as a review of historical and ethnographic sources. The symbols found at Kumbe Mayo revolve around, as seen materialized in the construction of the canal, narratives related to the sacredness of water, space-time, ancestors, and fertility. Furthermore, in the figures embodied in the caves, zoomorphic, geometric and anthropomorphic symbols are prevalent. These narratives extend to other sites in the central Andes and Mexico.
\end{abstract}

KeYworDs: caves, petroglyph, worldview and narratives. 


\section{Introducción}

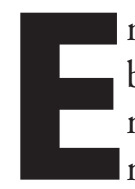
n la cosmovisión andina, las cuevas son simbólicamente el espacio mediador entre el mundo de aquí y de ahora (kaypacha) y el mundo interior (ukupacha), debido a que a través de ellas se ingresa a este último. Son concebidas como un lugar en el que habitan los ancestros; es decir, el espacio donde se gesta la vida (Polia, 1999: 168 y 169).

En este sentido, las diversas sociedades que se asentaron en los Andes centrales estuvieron vinculadas, desde tiempos tempranos, con las cuevas y los abrigos rocosos. Tal fue su importancia, que les dieron múltiples significados y usos, debido a que las concibieron como un lugar de origen, memoria, rito, habitacional, taller de herramientas y lugar de descanso final (machay) (Manzanilla, 1994: 59).

Son vastas las evidencias del uso de estos espacios en el territorio, así como su valor simbólico dentro de la cosmovisión andina, en el cual se manifestaron una serie de narrativas vinculadas con el culto a sus deidades, la organización del espaciotiempo, su relación con la naturaleza, su estar en el mundo, entre otros.

Al respecto, uno de los diversos complejos arqueológicos que albergan las cuevas ${ }^{1}$ como elemento simbólico es el centro ceremonial de Kumbe Mayo, ubicado en el actual departamento de Cajamarca, Perú (Fig. 1), a una altitud de $3600 \mathrm{~m} \mathrm{s.} \mathrm{n.} \mathrm{m,} \mathrm{en} \mathrm{el}$ que destacan cerros con laderas de pendientes suaves (Altujoyoj, Concejo, Lluspikaga, etc.), zonas semiplanas, afloramientos rocosos, quebradas (Torobamba, Secsemayo, entre otras), así como un río denominado Kumbe Mayo (Fig. 2).

En este contexto geográfico se encuentran los elementos arquitectónicos, entre los que destacan un canal esculpido en roca volcánica asociado a petroglifos, volúmenes pétreos (ubicados a lo largo del canal), muros y cuevas que fueron modificadas; precisamente, estas últimas serán analizadas en el presente artículo (Fig. 3).

1 En Kumbe Mayo se utiliza el nombre de cuevas en lugar de abrigos rocosos, debido a que tanto los investigadores como los visitantes las denominan así. No obstante, sus características implican que serían abrigos rocosos.
La construcción y ocupación inicial del centro ceremonial data de los periodos Formativo Medio y Tardío (1000 a. C.-550 a. C.), asociada a la fase Huacaloma Tardío, la cual se caracteriza por presentar sociedades autónomas con líderes de poderes restringidos (Seki, 2014: 193 y 194).

El uso del centro ceremonial se prolongó hasta la tradición Cajamarca (50 a. C.-1532 d.C.). No obstante, en este tiempo de larga data aún se desconoce el tipo de ocupación que se realizó en Kumbe Mayo.

En ese sentido, el propósito de este estudio es realizar un análisis de las cinco cuevas ubicadas alrededor del canal durante el periodo Formativo, análisis que buscará identificar las narrativas que se encuentran en dichos espacios.

\section{Antecedentes}

Las investigaciones sobre pinturas rupestres y petroglifos, para el periodo Formativo, fueron realizadas por Cardich (1964) en el área de Lauricocha-cuevas de Ranracancha 1-4. Al respecto, el investigador llegó a plantear seis estilos (A-F), de los cuales los $\mathrm{C}$ y $\mathrm{D}$ se vincularían con el periodo Formativo, pues se caracterizan por tener motivos de figuras esquematizadas de humanos, felinos y ofidios (Cardich, 1964: 137 y 141).

Por otra parte, Núñez Jiménez (1986) fue pionero en hacer un registro de los petroglifos correspondientes a diferentes periodos y lugares del territorio peruano, estudio que incluyó a los petroglifos del periodo Formativo. En esa misma línea, Guffroy (2011) hizo un estudio macro de los petroglifos y planteó tres tradiciones ( $\mathrm{A}, \mathrm{B}$ y $\mathrm{C}$ ) evolutivas; de ellas, sostuvo que la tradición A pertenecía al periodo Formativo, el cual se caracteriza por la presencia de seres sobrenaturales antropomorfos, en los que destacan aves y serpientes, y otros con motivos felínicos (Guffroy, 2011: 79). Sobre el particular, se puede advertir que la visión holística de Núñez y Guffroy se diferencia de los estudios de caso, como los realizados por Julio Abanto y Martín García (2007) en Cerro Cantería; Martín Mac Kay, Raphael Santa Cruz y Martín del Carpio (1999) en Poro Poro-Cajamarca; Quirino Olivera (2012) en Amazonas y Cajamarca; Daniel Castillo Benítez y María S. Barrau (2014) en el Alto de la Guitarra, y Gordon Ambrosino (2019) en la 


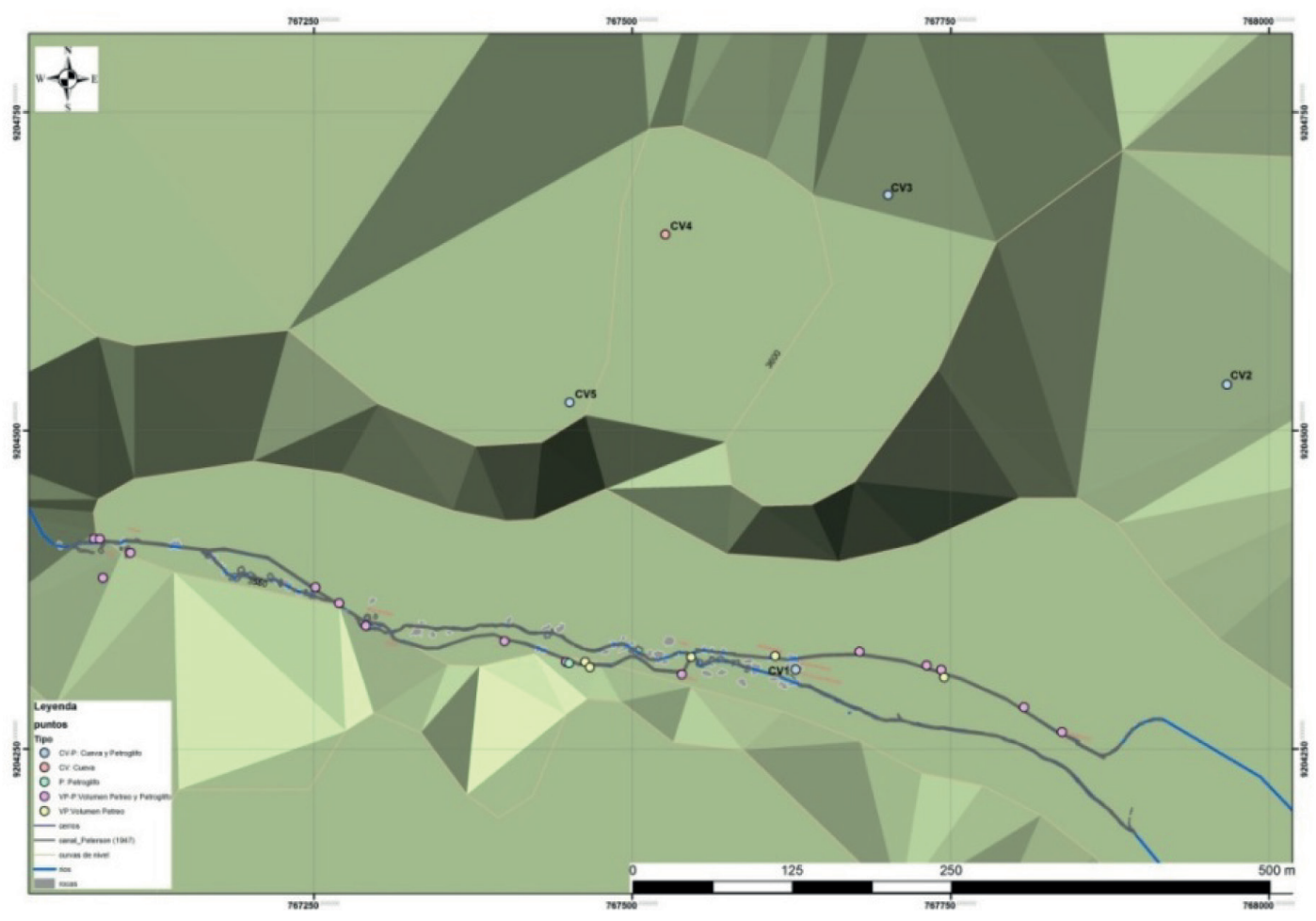

Figura 3: Plano del primer tramo del canal que incluye los volúmenes pétreos, petroglifos y cuevas Fuente: Basado en Tello (2004), Petersen (1969) y Jones (2010)

Una década posterior, Silva y Leca (1997) realizaron excavaciones en la zona de La toma y en las áreas adyacentes al canal.

Posteriormente, Ignacio Alva (2006) realizó una prospección en el área de Kumbe Mayo, trabajo que incluyó el registro de las cuevas 1 y 2 . A tal efecto, llegó a sostener que las figuras plasmadas en la cueva 2 se tratarían de un paisaje montańoso conformado por la fauna, flora y los accidentes geográficos (Alva, 2006: 61 y 62).

Otro de los estudios realizados en el área es el de Kimberly Lynn Jones (2010), cuando analizó el corpus visual Cupisnique. La investigadora planteó que este sistema ideológico se extendió y fue adoptado en la cuenca de Cajamarca y, por ende, en Kumbe Mayo. De este corpus de imágenes, Jones estableció temas generales vinculados a los conceptos de captura-decapitación, fertilidad y dualidades simbólicas (Jones, 2010: 271).

Asimismo, la especialista señala que la iconografía Cupisnique se encuentra inmersa dentro de una ecología ritual en la que destacan determinadas plantas y animales (felinos, serpientes, monos, ciervos, ranas, roedores, entre otros). De las evidencias anteriores, los conceptos identificados por la autora aluden, a su vez, a un sistema de dualidades simbólicas, como son caza-sacrificio, sacrificio-fertilidad, rojo-negro, mujer-hombre, izquierda-derecha, sangre-agua, etc. (Jones, 2010: 273).

Lo tratado en los párrafos anteriores muestran los aportes al estudio de Kumbe Mayo, pero también generan múltiples interrogantes, como la siguiente: ¿Qué narrativas se encuentran en las cuevas de Kumbe Mayo?

\section{La cosmovisión en los Andes centrales y las cuevas de Kumbe Mayo}

La cosmovisión para los pobladores de los Andes centrales «tiende a situar la experiencia humana a escala cósmica, entendiendo que discurre en permanente relación y co-operación [sic] con los otros seres que componen el cosmos y que, al igual que el hombre, constituyen comunidades de vida vinculadas entre sí» (Depaz, 2015: 21).

En esa forma de concebir el mundo, compartían nociones y principios vinculados con el espacio y el tiempo, comprendidos como una unidad y no como algo separado. Esto se ve en la definición del concepto pacha en aymara, definido usualmente como tiempo o tiempo antiguo, pero también se lo asocia al cielo, a 
la tierra y al infierno (Bertonio, [1612] 2008: segunda parte [242]). Aunado a ello, en el quechua se da la división de los espacios temporales, donde el kay pacha alude espacialmente al centro y al lugar de encuentro entre el hombre y sus deidades, y temporalmente se relaciona con el aquí y el ahora. Otro de los espacios temporales es el hanaq pacha, que se vincula con el espacio de las deidades y los astros; mientras que el uray o ukhu pacha hace referencia al mundo interior (que representa el lugar de los muertos) y se relaciona con el lugar donde se gesta la vida (Sherbondy, 1987: 186; Earls y Silverblatt, 1976: 300; Estermann, 1998: 158; Polia, 1999: 159 y Paz, 2015: 198).

Por ello los cerros, el agua, los ancestros o las plantas, entre otros, eran considerados sagrados, lo cual se vincula con el concepto de waqa, que viene a ser la potencia, el exceso a lo rutinario, dado que posee esa dualidad de ser peligroso o de ser bueno e inofensivo, así como de estar presente y oculto (Depaz, 2015: 152 y 153). En esta perspectiva de ver el mundo, los diversos elementos que formaban parte del entorno eran poseedores del kamaq o sami, concepto que alude a la fuerza vital, dando así la misma esencia a todos los seres, pero con su propio atributo.

En este escenario, las sociedades comunicaron y plasmaron sus saberes sobre las paredes de las cuevas, arquitectura, cerámica, textiles, entre otros, llegando así a perennizar y materializar dichos saberes.

Este es el caso del centro ceremonial de Kumbe Mayo, en el que sobre las paredes de las cuevas, el canal y los volúmenes pétreos se realizaron una serie de símbolos, considerados "formulaciones tangibles de ideas, abstracciones de la experiencia fijadas en formas perceptibles, representaciones concretas de ideas, de actitudes, de juicios, de anhelos o de creencias» (Geertz, 2003: 90). De ahí que los símbolos de Kumbe Mayo dan cuenta de las narrativas que se quería trasmitir.

De manera que los símbolos hallados en Kumbe Mayo tenían sentido y eran comprendidos por todos aquellos que conocían a qué elementos sagrados evocaban, así como el significado que tenían dentro de su cosmovisión.

\section{Metodología del análisis de los símbolos}

El análisis partió de un estudio espacial de la ubicación de las cuevas, en relación con el canal, para reconocer patrones y diferencias en cuanto a su disposición.

Seguidamente, se realizó un estudio respecto a la técnica del dibujo, el cual incluyó el grosor de la línea, la profundidad y la forma.

A nivel interpretativo, el estudio se ha basado en los datos biológicos de las especies y sus hábitats, así como en la arqueología, historia y etnografía, y se relacionan con los símbolos vinculados a la sacralidad del agua, el mundo interior, los ancestros y la fertilidad.

\section{Los elementos arquitectónicos de Kumbe Mayo}

A continuación, se describe brevemente el canal, los volúmenes pétreos y muros, para luego centrar la atención en las cuevas, que son el motivo del presente artículo.

\section{El canal y los volúmenes pétreos}

El inicio visible del canal se ubica en el sitio denominado La toma, punto en el que también se encuentra el río (de nombre topónimo) que se origina al pie del cerro Kargashkunga, donde es alimentado por varias lagunillas, entre las que destaca Torobamba (Tello, 2004: 235). En este recorrido, el canal presenta un primer tramo esculpido en roca volcánica y un segundo tramo elaborado en tierra y roca que desciende por las laderas de los cerros hasta desembocar en un reservorio que se ubica al pie del cerro Apolonia (Petersen, 1985: 98).

El canal esculpido en roca volcánica tiene una longitud de $850 \mathrm{~m}$, con 35 a $50 \mathrm{~cm}$ de ancho y 30 a $65 \mathrm{~cm}$ de profundidad (Petersen, 1969: 114). Es un tramo en el que el canal adopta formas de tipo escalonada, recta y curva, y sus paredes contienen una serie de símbolos con formas geométricas, partes del cuerpo (plantas de pies), cruces cuadradas y líneas serpenteantes, escalonadas o zoomorfas, entre otros. En este primer tramo, el canal tiene una alternancia con el río, debido a que inicialmente se encuentra en la margen izquierda, para luego cambiar a la derecha y volver a la izquierda.

El segundo tramo, construido en tierra y roca, tiene una longitud de $8220 \mathrm{~m}$, cubriendo así una longitud mayor del canal. En ese tramo el canal cru- 
za la divisoria continental y traslada así el agua de la vertiente del océano Pacífico al océano Atlántico (Petersen, 1985: 97 y 98). Asimismo, en este trayecto el canal se encuentra próximo al centro ceremonial de Layzón y al sitio de Agua Tapada, para luego desembocar en Santa Apolonia.

\section{Estructuras cerca de La toma}

En la margen derecha del canal, en el denominado sector de La toma, se ubican muros pequeños elaborados en roca volcánica. Los muros poseen un acabado fino y de similar forma que la construcción del canal, en el cual también se encuentra un petroglifo con diseños geométricos. No obstante, debido a que las excavaciones fueron restringidas, no se conoció qué tipo de lugar era y tan solo se planteó que se trataría de estructuras relacionadas a aspectos ceremoniales (Silva y Leca, 1997: 15).

\section{Las cuevas de Kumbe Mayo}

La disposición de las cuevas en Kumbe Mayo giran en torno al canal; la cueva 1 se ubica al sur, mientras que las 2, 3, 4 y 5 se ubican al norte (Fig. 3).

En cuanto a las distancias de las cuevas con el canal, varían desde $8.62 \mathrm{~m}$ hasta $360.29 \mathrm{~m}$, aproximadamente, en línea recta; mientras que la distancia entre las cuevas tiene una mínima de $177.11 \mathrm{~m}$ y una máxima de $405.83 \mathrm{~m}$.

Respecto a la forma de acceso, la primera cueva presenta forma triangular y cuatro escalinatas; a diferencia de la segunda cueva, que tiene forma ovalada y base plana, con siete plataformas y techo abovedado. Asimismo, las cuevas que no presentan plataformas para acceder son de forma irregular, rectangular y ovalada de techo plano. Además, las cuevas 1, 2 y 5 poseen petroglifos en sus paredes; la cueva 3 posee volúmenes pétreos con tallas y la cueva 4 no presenta ningún petroglifo.

\section{Análisis iconográfico de los símbolos hallados en las cuevas}

Cueva 1. Los símbolos plasmados en la parte central, en el frontis y al lado derecho de la cueva fueron elaborados a partir de la técnica del bajo relieve de línea gruesa (Fig. 4).

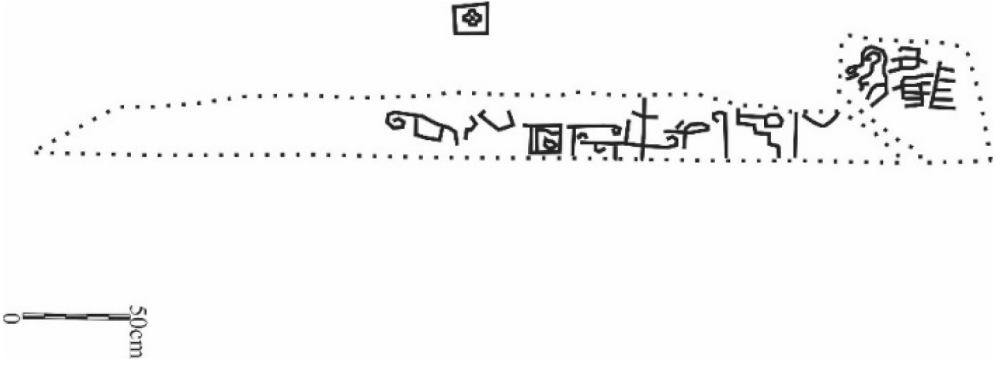

Figura 4: La cueva 1

Fuente. Redibujado de Jones (2010, p. 382)

La composición de las figuras tiene como eje a una cruz cuadrada, la cual se ubica en la parte interna y central de la cueva. Esta figura también se encuentra dentro de un cuadrado y contiene en la parte media a un círculo.

Hacia el lado externo de la cueva, en el frontis, la composición está conformada por líneas verticales, escalonadas, horizontales y curvas; algunas de las cuales llegan a intersectarse. Un elemento adicional que tienen estas líneas es que culminan en círculos o volutas. A las figuras mencionadas, debajo de la cruz se encuentra un cuadrado, así como líneas irregulares.

Al lado derecho del frontis descrito, se localiza otra composición constituida por figuras geométricas de forma cuadrangular, así como líneas ondulantes e irregulares.

Cueva 2. Se ubica en la parte baja de un afloramiento rocoso. Visto de lado, adopta la forma de un perfil

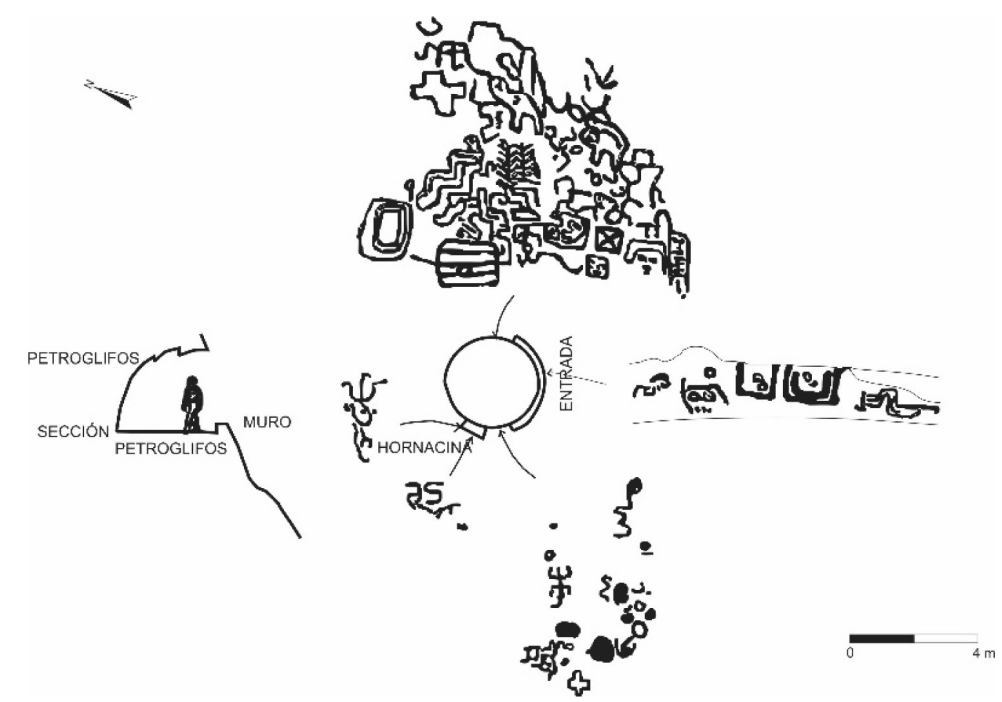

Figura 5: Símbolos de la cueva 2 Fuente. Basado en Núñez (1986; l: 300). 
humano. El ingreso a la cueva tiene la apariencia de una boca. En la parte interna presenta forma abovedada y sus paredes muestran símbolos elaborados con la técnica del bajo relieve, que alternan líneas gruesas y delgadas. La pared derecha muestra figuras antropomorfas, geométricas y zoomorfas (Fig. 5); al lado izquierdo, una hornacina con símbolos también geométricos y zoomorfos, y el piso posee horadaciones.

La disposición de los símbolos se encuentra organizada espacialmente. En la zona central de la pared derecha hay dos figuras a modo de corchetes, uno frente al otro; y de su parte superior, salen dos arbustos. Hacia el lado inferior derecho, se encuentran las figuras antropomorfas ubicadas de frente, una de ellas con un cuerpo esqueletizado (con un apéndice que sale de su cabeza) y las otras tres son cabezas, una de las cuales presenta los ojos vacíos. Aunado a ello, en el lado superior derecho, las figuras zoomorfas se encuentran de perfil y de forma ascendente, asociadas a pequeńos círculos; mientras que las figuras serpentiformes están presentes en casi todo el panel. La figura de la cruz cuadrada se encuentra hacia el lado superior izquierdo; en la parte inferior izquierda, se ubican figuras escalonadas, cuadrados concéntricos y una figura cuadrangular que contiene líneas horizontales.

Al lado izquierdo de la cueva, se ubica una hornacina, y en su pared derecha se halla la imagen de una rana esquematizada, orientada hacia abajo; a su vez, esta figura se encuentra sobre dos líneas ondulantes. En la parte central de la hornacina, se realizaron dos líneas con forma de $S$; y debajo de ellas, se plasmó la imagen de un ser zoomorfo en posición de perfil. Entre tanto, al lado izquierdo las imágenes son líneas serpenteantes, figuras lagartiformes, círculos y una cruz cuadrada, en la cual las figuras zoomorfas se encuentran orientadas hacia la parte superior.

Otro de los espacios en los que se plasmaron las figuras fue en la parte interna del frontis de la cueva, en cuya zona central se tallaron algunas $U$ cuadradas que contienen círculos. Hacia el lado derecho, se tallaron líneas escalonadas; al lado izquierdo de la composición, se realizaron líneas irregulares, rectas, oblicuas, curvas y círculos. Asimismo, en el piso de la cueva se plasmaron círculos de tamaño mediano y pequeño, así como líneas irregulares en bajo relieve.
Cueva 3. Presenta un acceso irregular. En su parte interna y externa posee dos volúmenes pétreos con símbolos de incisos, elaborados con la técnica del alto y bajo relieve con un tipo de línea gruesa.

El volumen pétreo que se ubica al interior de la cueva tiene forma cónica y está ubicado de manera frontal; además, muestra incisiones profundas y gruesas. La figura presenta un contorno irregular $\mathrm{y}$ en la parte superior posee dos figuras ovaladas a modo de ojos; mientras que en la zona inferior, se plasmaron líneas horizontales paralelas, en cuya parte interna se realizaron figuras serpenteantes orientadas hacia arriba, a la derecha e izquierda. Además, presenta líneas escalonadas, y al lado izquierdo posee figuras circulares, tanto en la parte interna como al exterior de la figura. Se trataría, entonces, de un ser zoomorfo (Fig. 6).

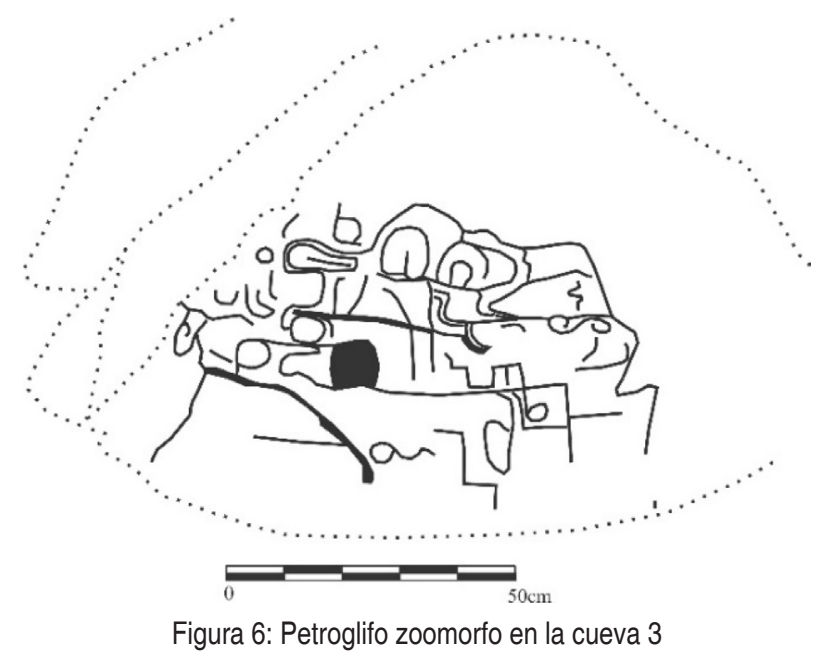

El volumen pétreo, ubicado en la parte externa de la cueva, presenta forma alargada y un extremo redondeado que adquiere la apariencia de una cabeza. En la parte frontal se plasmaron dos líneas paralelas, y en el espacio que hay entre ambas, se realizaron incisiones en forma de colmillos. Hacia la zona de la frente, se encuentran líneas serpenteantes, entre otras; al lado izquierdo de la cabeza, así como del cuerpo, se halla una línea doble en forma de L, de la que cuelgan colmillos; y al lado derecho, un círculo. En la parte superior de la cabeza y del cuerpo, se dibujaron círculos, líneas en forma de $S$ y líneas serpenteantes (Fig. 7). En conjunto, los motivos que forman parte del volumen pétreo tienen la estructura de un felino. 


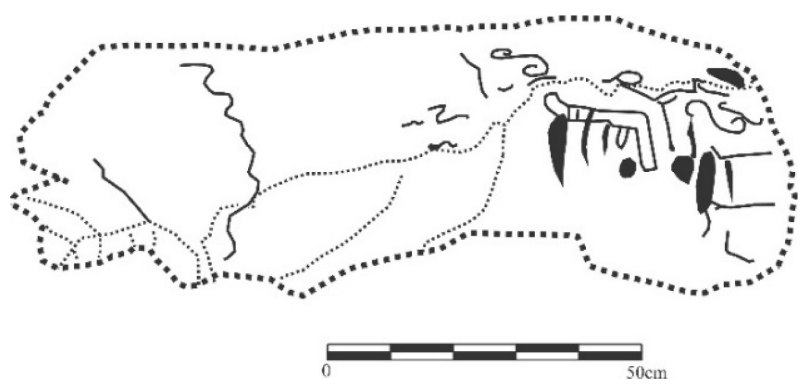

Figura 7: Petroglifo con la forma de un felino en la cueva 3

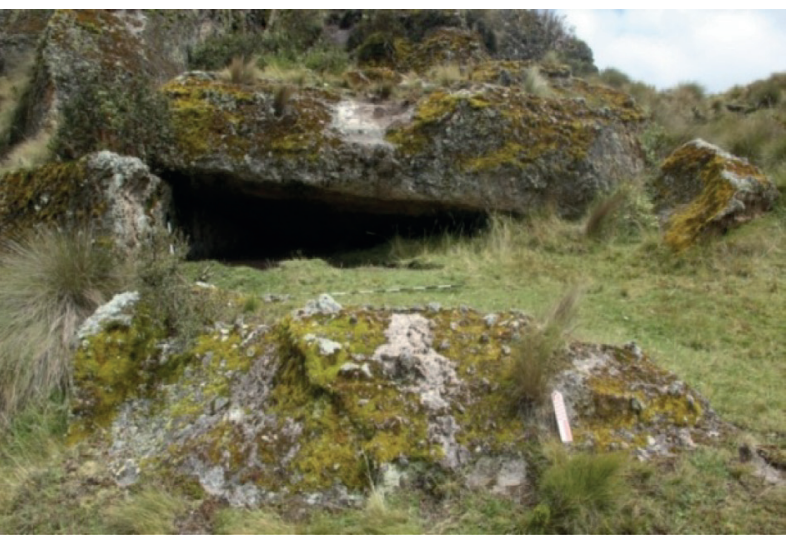

Figura 8: Vista panorámica de la cueva 4

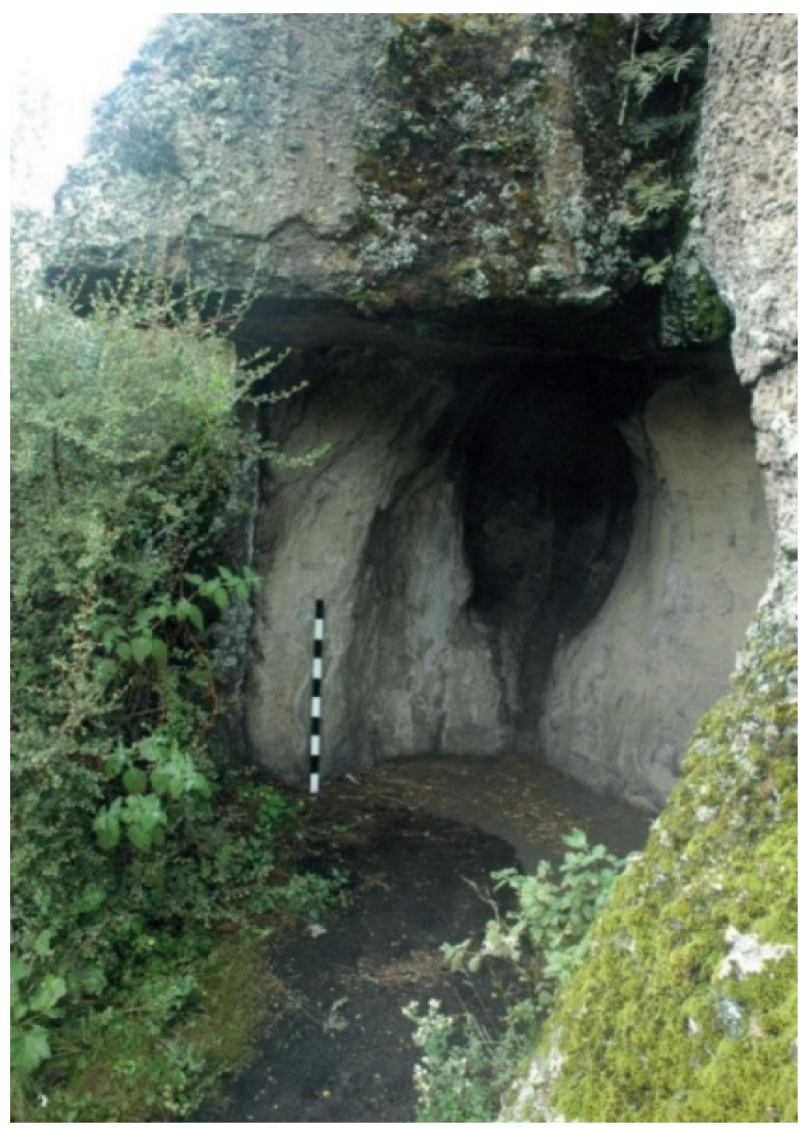

Figura 9: Vista de la cueva 5
Cueva 4. Presenta un ingreso de forma rectangular. Su techo está elaborado con una piedra que descansa por un lado sobre otra que tiene forma cuadrangular; ambas piedras fueron cortadas y pulidas, pero no poseen figuras (Fig. 8).

Cueva 5. Posee un ingreso de apariencia ovalada, con un techo plano que fue tallado y cortado. En la parte interna, tiene otra horadación con la forma de una vagina, debajo de la cual se encuentran las figuras elaboradas con la técnica del bajo relieve de un tipo de línea gruesa (Fig. 9).

La composición presenta una línea serpenteante y debajo, una figura curva y formas ovaladas. Adicionalmente, sobre la línea se encuentra otra figura ovalada (Fig. 10).

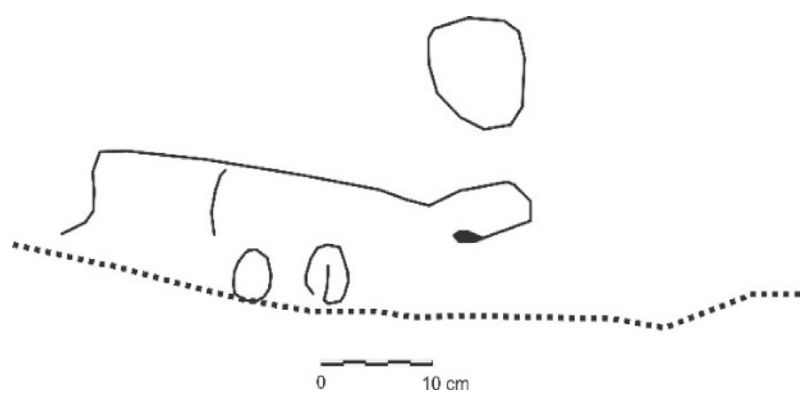

Figura 10: Petroglifo en la cueva 5.

\section{Resultados}

La elección de los afloramientos rocosos en los que fueron esculpidas las cuevas se basó en la asociación que tenían con el primer tramo del canal, que alberga mayoritariamente a los petroglifos. En dicha disposición espacial no hay una regularidad en cuanto a la distancia entre las cuevas, ya que cuatro de ellas se encuentran al norte y una en el sur. Asimismo, no hay una simetría en la distancia.

En lo que respecta a la técnica de talla, se han podido establecer dos estilos. El primero se encuentra en las cuevas 1, 2 y 5, que fueron realizadas en bajo relieve, de incisiones profundas, con representaciones de figuras antropomorfas orientadas de manera frontal y figuras zoomorfas ubicadas de perfil. El segundo estilo se caracteriza por estar elaborado en alto relieve, con incisiones profundas y de mayor grosor, estilo que se halló en las cuevas 3 y 5 . 
Las cuevas analizadas en Kumbe Mayo fueron espacios culturizados, ya que se modificaron con el propósito de incluirlos dentro de una planificación arquitectónica mayor, en donde el canal fuera el eje central. Adicionalmente, los elementos arquitectónicos y los petroglifos formaron parte de esta planificación, como elementos simbólicos que dieron cuenta de las narrativas que buscaban trasmitir en el centro ceremonial.

Los símbolos asociados a las cuevas de Kumbe Mayo giran en torno a la narrativa de la sacralidad del agua, marcada por su relación con el canal, el río y los petroglifos del canal. A razón de que el río y el canal, en la cosmovisión de los pobladores de los Andes centrales, son los lugares por donde fluye el sami ${ }^{2}$, a modo de un sistema circulatorio que lleva el agua a todo el cosmos (Allen, 2008: 60).

Esta narrativa se asocia con los símbolos del agua y la humedad, que se encuentran en las cuevas 2, 3 y 5 , donde se hallan figuras serpenteantes y de ranas. Las primeras figuras se relacionan por la forma que adquieren con los ríos; las segundas, de acuerdo con sus características biológicas, se caracterizan por habitar en espacios húmedos, ya que pasan la mayor parte del tiempo en el agua. Especies que en las sociedades de los Andes centrales, dada su relación con su hábitat, su aparición en las estaciones de invierno, la mitología y la iconografía, son vinculadas con el agua y la fertilidad (Polia, 1999: 185; Carrión, 2005: 27; Golte, 2009: 174 y Jones, 2010: 232).

Otro de los símbolos asociados con el agua es el felino, que se ubica en la cueva 3 y presenta motivos serpenteantes y círculos en su cuerpo. Esta relación del felino con el mundo interior y el agua se encuentra desde el periodo Formativo hasta el Horizonte Tardío, etapa en la que fue el elemento simbólico que representó la fuerza vital del mundo interior (Fujii, 1993: 272 y 273).

Otra de las narrativas que se buscó trasmitir en las cuevas es la concepción del tiempo y el espacio, marcado por la cueva como comunicador entre el ukhu pacha y el kaypacha. Asimismo, la presencia de los círculos, espirales y la cruz cuadrada son representaciones del tiempo, no como algo lineal sino discontinuo, que está formado por etapas que son interrumpidas por tiempos de destrucción que se renuevan. Asimismo, la cruz cuadrada alude a la cuaternidad del

2 Sami es también conocido como la fuerza vital. espacio, que involucra la organización del tiempo y el espacio basado en los puntos cardinales que llegan a conectar con el ukhu pacha y kaypacha (Esterman, 1998: 166 y 183; Allen, 2008: 75; Martínez, 2010: 166 y 167).

Siguiendo con los discursos, se encontró que la cueva 2 buscó trasmitir la presencia del ancestro, materializado en el perfil humano que se observa en el farallón. En el interior de la cueva, que tiene la forma de una boca, se plasmaron las figuras del esqueleto y las cabezas antropomorfas, que remiten a los mitos de las deidades y de héroes civilizadores y ancestros como Kuan en Cajamarca; Cuniraya Viracocha, Pariacaca y Collquiri en Huarochirí; Guari y Libiac en Cajatambo; los wamanis en Ayacucho; Wiraqocha e Inca Roca en el sur. Al ser las entidades encargadas de la construcción de los primeros canales, chacras, reservorios y casas, estaban facultados de dar y controlar el agua o la comida, y de generar el bienestar de los pueblos (Arguedas, 1975; Randall, 1987; Sherbondy, 1987; Polia, 1999; Duviols, 2003; Tello, 2004; Millones, 2013, entre otros).

La sacralidad del agua y los ancestros dadores de estos elementos aluden a la fertilidad, debido a que se trata de sociedades agrícolas que se reflejan en las cuevas como lugares donde se gesta la vida. Asimismo, el agua del río y del canal actúan como fecundadores de la tierra, y los símbolos de las cuevas 2 y 5 , como las plantas, las ranas y la vagina, aluden a la fertilidad y la abundancia.

En tal sentido, el corpus simbólico hallado en Kumbe Mayo da cuenta de la cosmovisión de sus líderes y de las sociedades que formaban parte de él. El centro ceremonial fue considerado un lugar donde se formalizaron y legitimaron los símbolos asociados con ideas y conceptos (que tenían que ver con el ordenamiento del tiempo y del espacio), así como el origen y la adjudicación del canal por el cual se trasladaba el agua para las actividades vinculadas a aspectos ceremoniales y funcionales, que se diferenciaban polisémicamente a través de los símbolos que se interconectaban en su recorrido.

\section{Reflexiones finales}

Las narrativas que se buscaron trasmitir en Kumbe Mayo se extienden a otros lugares de los Andes cen- 
trales. La diferencia se halla en el contexto en el que se encuentran, como son los elementos arquitectónicos asociados y la composición de los paneles.

Así tenemos lugares abiertos, como pampas, zonas de desierto; quebradas y farallones como Quebrada de los Boliches-Olmos (Lambayeque), Cerro Mulato-Chongoyape (Lambayeque), El Pongo (Cajamarca), Pay Pay-Contumazá (Cajamarca) y el sitio de Yonán, también ubicado en Contumazá, Pampa del Castillo (La Libertad, Trujillo), Alto de la Guitarra (La Libertad), Palamenco-Santa (Áncash) y Huancor (Chincha, Ica), donde se han identificado petroglifos en los que se han plasmado cruces cuadradas, figuras geométricas, antropomorfas (cabezas) y zoomorfas (líneas serpentiformes, lagartiformes y ranas esquematizadas) (Núñez, 1986: I, II y III) que presentan la misma técnica y forma que las registradas en Kumbe Mayo.

En cuanto a contextos de cuevas sin asociaciones arquitectónicas, pero con símbolos similares a nuestra área de estudio, tenemos a San Miguel, Cajamarca, espacio en el cual se encuentra un panel que presenta también figuras geométricas (cuadrados y círculos simples, así como cuadrados y círculos concéntricos o con un punto al interior, escalonados, espirales y volutas), antropomorfas (cabeza) y zoomorfas (ranas, líneas serpentiformes y felinos) (Núñez, 1986; I: 173).

Otra de las cuevas que guarda una estrecha relación con Kumbe Mayo es la de Kiñan Tanka, la cual se asocia a un canal tallado en piedra. Los símbolos plasmados en esta cueva, y definidos como estilos 1 y 2, se relacionan con las figuras definidas en Kumbe Mayo como estilo 1, en las que destacan figuras antropomorfas ubicadas de frente y seres zoomorfos orientados de perfil.

Ambrosino seńala que la cueva de Kiñan Tanka habría sido un lugar ceremonial y de peregrinaje al cual acudieron individuos de diversos lugares, quienes realizaron las inscripciones buscando vincularse con los que habían estado ahí antes, dándole continuidad a las narrativas, pero también para transformarlas y diversificarlas. (Ambrosino, 2019: 150).

En Poro Poro, Cajamarca, también encontramos lugares con pinturas rupestres, petroglifos, pozos y canales. Sitio en el que los símbolos hallados tratan del culto a las divinidades, así como su relación con el agua y el clima (Mac Kay et al., 1999: 397). Esta relación de los símbolos en los petroglifos también se encuentra en Cerro Cantería; sin embargo, en este caso, el culto vendría a ser al cerro tutelar (Abanto y García, 2007). Asimismo, las poblaciones en Alto de la Guitarra habrían peregrinado al lugar, con el fin de solicitar y agradecer a los apus tutelares (cerros) y a los ancestros míticos (plasmados en los petroglifos) por su acceso al agua, por la fertilidad de sus animales y el rendimiento de sus producciones agrícolas (Castillo y Barrau, 2014: 309, 318 y 319).

Los sitios mencionados, al igual que Kumbe Mayo, habrían sido marcadores del espacio, poseedores de la fuerza vital y lugares de peregrinaje, en los cuales se habrían realizado rituales (en rememoración a los tiempos míticos) y en donde se trasmitían narrativas. Un paralelo con ello sería la forma en que los pobladores de Sonqo conciben su territorio:

Un paisaje de constelaciones topográficas familiares que sirven como puntos de referencia, tanto en el espacio como en el tiempo. Para los runakuna, estos rasgos topográficos son lugares sagrados llamados tirakuna y los consideran como una sociedad paralela a la de ellos, compuestas por poderosas personalidades animadas. (Allen, 2008: 34)

Los símbolos hallados en las cuevas de Kumbe Mayo también permiten hacer paralelos con el caso de las cuevas de México. Silvia Limón ya había realizado un amplio trabajo del caso Inca y Mexica, desde el punto de lugar de origen. A continuación, el paralelismo se abordará desde la conceptualización de la cueva, relacionada con el mundo interior y los símbolos presentes.

Dentro de la cosmovisión de los pueblos originarios de México, «la cueva es la entrada al inframundo (y, por lo tanto, una cámara funeraria), pero también es acceso al vientre de la tierra a la boca del monstruo terrestre. Por extensión, es sitio donde la fertilidad puede ser propiciada» (Manzanilla, 1994: 59 y 60). Esta conceptualización de la cueva es semejante a las sociedades del área andina, debido a que también representa el ingreso al mundo interior $\mathrm{y}$, sobre todo, al espacio donde se gesta la vida, la fertilidad. Un ejemplo de ello son los símbolos plasmados en la cueva 2, donde se representa al ancestro, la flora y la fauna de ese mundo interior, así como en la cueva 5, en la cual se esculpió la figura de una vagina.

Otro punto de encuentro entre las sociedades de México y los Andes es que las cuevas se relacionan 
con la sacralidad del agua, debido a los símbolos que presentan. Tal es el caso de Totemihuaca, Puebla, en donde se encontró un túnel trabajado con presencia de figuras de ranas, el cual podría estar aludiendo al culto al agua (Manzanilla, 1994: 60). Este símbolo de la rana, vinculado a la sacralidad del agua, fue ampliamente registrado en diversos soportes culturales de las sociedades de los Andes centrales, del cual Kumbe Mayo no sería la excepción.

Asimismo, los mitos relacionados al hecho de que las deidades eran los dadores y controladores del agua se repiten en ambas áreas. Como señala el cronista Francisco de Burgoa, eran entidades que tenían el control del agua y de las semillas, así como de los que habitaban en aquellos espacios (Manzanilla, 1994: 60). Estos mitos tienen relación con los símbolos hallados en Kumbe Mayo, lugar en el que se buscó simbolizar la presencia del ancestro.

\section{Conclusiones}

Las narrativas que se buscaron trasmitir en las cuevas de Kumbe Mayo giran en torno a la sacralidad del agua, manifestada en la vinculación de las cuevas con el canal y con los petroglifos que se encuentran a lo largo, a modo de estaciones. Asimismo, las figuras serpentiformes, las ranas, las figuras lagartiformes y el felino son animales asociados a la humedad, estaciones de invierno, así como la mitología e iconografía, y han sido vinculados desde tiempos ancestrales al agua. Otra de las narrativas que se buscó trasmitir fue la del tiempo y el espacio, la cual fue marcada por la cueva como el ingreso al mundo interior (ukupacha), y por la presencia de los símbolos del círculo, el espiral y la cruz cuadrada. En esta misma línea, se fijó en la memoria la presencia del ancestro como dador del agua, lo cual era una manera de rememorarlo. La fertilidad es otra de las narrativas que se halla en el agua del canal y del río, como símbolos fecundadores de la tierra, y donde las plantas, animales, ranas y la vagina son símbolos de abundancia y fertilidad.

Las narrativas halladas en Kumbe Mayo no se restringen a esa área, sino que se extendieron a otros lugares. Estos espacios habrían sido lugares de rito que pudieron funcionar como marcadores en el espacio, en el que se rememorarían tiempos míticos y se trasmitían narrativas vinculadas a la sacralidad del agua, el espacio-tiempo, los ancestros, la fertilidad, entre otros, manteniéndose a lo largo del tiempo y transformándose con los cambios propios de los contextos y procesos sociales.

\section{Agradecimientos}

El presente estudio ha sido posible gracias a la guía del Dr. Luis Millones, quien con su confianza y enseñanza me alentó a continuar el trabajo en Kumbe Mayo. Asimismo, quiero agradecer a Iván Díaz Lon por ayudarme con algunos de los gráficos presentados en este artículo.

\section{Referencias citadas}

Abanto, J. y García-Godos, M. (2007). Los petroglifos formativos de cerro Cantería. Un adoratorio temprano entre la cuenca alta de quebrada Canto Grande y quebrada El Progreso, Lima. Arkeos. https://rupestre. equiponaya.com.ar/articulos/petroglifos_cerro_canteria.htm

Ambrosino, G. (2019). Inscription, place and memory: palimpsest rock art and the evolution of highland, andean social landscape in the formative period (1500200BC), H-ART. Revista de historia, teoría y crítica de arte, (5), pp. 127-156.

Arguedas, J. M. y Duviols, P. (1966). Dioses y hombres de Huarochiri. Narración quechua recogida por Francisco de Ávila [¿1598?]. Lima: Instituto de Estudios Peruanos.

Auva, I. (2006). Kumbe Mayo: Centro ceremonial formativo de culto al agua y paraje sagrado en Cajamarca. [Tesis de licenciatura]. Departamento de Ciencias Sociales, Universidad Nacional de Trujillo, Trujillo.

Allen, C. J. (2008). La coca sabe. Coca e identidad cultural en una comunidad andina (Trad. L. León). Cusco: Centro Bartolomé de las Casas.

Bertonio, L. (2008) [1612]. Vocabvlario de la Lengua Aymara. Editorial Botello, Instituto de Estudios Bolivianos (IEB) y Team Bolivia Bilateral Research Cooperation.

Carrión, R. (2005). El culto al agua en el antiguo Perú. Lima: Instituto Nacional de Cultura.

Cardich, A. (1964). Lauricocha. Fundamentos para una prehistoria de los Andes Centrales (Vol. III. Studia 
Praehistórica). Buenos Aires: Centro Argentino de Estudios Prehistóricos.

Castillo, D. y Barrau, M. (2014). Arte rupestre en el Alto de la Guitarra: Paraíso de cazadores, calendario agrícola, mitología y ritual en la costa norte peruana. Conchopata, (4), 309-334.

Depaz, Z. (2015). La cosmo-visión andina en el Manuscrito de Huarochirí. Lima: Vicio Perpetuo/Vicio Perfecto.

Duviols, P. (2003). Procesos y visitas de idolatría: Cajatambo, Siglo XVII. Lima: Instituto Francés de Estudios Andinos, Fondo Editorial de la Pontificia Universidad Católica del Perú.

Estermann, J. (1998). Filosofía andina. Estudio intercultural de la sabiduría autóctona andina. Quito: Abya-Yala.

Earl, J. y Silverblatt, I. (1978). La realidad física y social en la cosmología andina. Actas del XLII Congrés International des Américanistes, (4), 299-325.

FujII, T. (1993). El felino, el mundo subterráneo y el rito de fertilidad: tres elementos principales de la ideología andina. Senri Ethnological Studies, (37), 259-274.

Geertz, C. (2003). La interpretación de las culturas. Barcelona: Gedisa.

Golte, J. (2009). Moche: cosmología y sociedad. Una interpretación iconográfica. Lima: Instituto de Estudios Peruanos, Centro Bartolomé de las Casas.

Guffroy, J. (2011). Las tradiciones centro-andinas de rocas grabadas (Perú): evoluciones y continuidades. Chungará, 43(1), 73-88.

Jones, K. (2010). Cupisnique culture: the development of ideology in the ancient Andes. [Tesis doctoral]. Universidad de Texas, Austin.

Limón, S. (2009). Las cuevas y mito de origen: los casos Inca y Mexica (2. ${ }^{\mathrm{a}}$ ed.). México: Centro de investigaciones sobre América Latina y el Caribe, Universidad Nacional Autónoma de México.

Mac Kay, M.; Santa Cruz, R. y Del Carpio, M. (1999). Arte rupestre en el sitio Formativo de Poro Poro, sierra norte del Perú. BIRA, (26), 393-402.

ManZanilla, L. (1994). Las cuevas en el mundo mesoamericano. Ciencia, (36), 59-66.

Martínez, C. (2010). De manera sagrada y en celebración. Identidad, cosmovisión y espiritualidad en los pueblos indígenas. Buenos Aires: Biblos.
Millones, L. (2013). Divinidades bajo las aguas. Lima: Apus Graph Ediciones.

Núñez, A. (1986). Petroglifos del Perú. Panorama mundial del arte rupestre. (Vol. 1- 4). La Habana: Unesco.

Olivera, Q. (2012). Arte rupestre en la cuenca del Marañón. Regiones de Amazonas y Cajamarca, Perú. Investigaciones Sociales, 16(28), 397-402.

Petersen, G. (1969). Cumbemayo: acueducto arqueológico que cruza la divisoria continental. Revista técnico cientifica, XI(3), 112-139.

Petersen, G. (1985). Cumbemayo: acueducto arqueológico que cruza la divisoria continental. En R. Ravines (Ed.), Historia de Cajamarca (pp. 97-100). Lima: Instituto Francés de Estudios Andinos.

Polia, M. (1999). La cosmovisión religiosa andina en los documentos del archivo romano de La compañia de Jesús (1581-1752). Lima: Fondo Editorial de la Pontificia Universidad Católica del Perú.

Randall, R. (1987). Del tiempo y del río. El ciclo de la historia y la energía en la cosmología incaica. Boletín de Lima, (54), 69-95.

Silva Vigo, E. y Lecca, C. (1997). Investigación, conservación y defensa del complejo arqueológico Cumbe Mayo. Cajamarca: Instituto Nacional de Cultura.

SEKI, Y. (1994). La transformación de los centros ceremoniales del Periodo Formativo en la cuenca de Cajamarca, Perú. En L. Millones \& Y. Onuki (Eds.). El Mundo ceremonial andino (pp.143-165). Lima: Horizonte.

SEKI, Y. (2014). La diversidad del poder en la sociedad del Periodo Formativo. Una perspectiva desde la sierra norte. En Y. Seki (Ed.). El centro ceremonial andino. Nuevas perspectivas para los periodos Arcaico y Formativo (Senri Etnological Studies 89) (175-200). National Museum of Ethnology.

Sherbondy, E. (1987). Organización hidráulica y poder en el Cuzco de los Incas. Revista Española de Antropología Americana (17), 117-153.

Tello, J. C. (2004). Arqueología de Cajamarca: Expedición al Marañon-1937. Lima: Fondo Editorial de la Universidad Nacional Mayor de San Marcos.

Williams, C. y Pineda, J. (1983). Arquitectura temprana en Cajamarca. Gaceta Arqueológica Andina, 1(6), 4-5. 